\title{
Effects of breeder age and early energy restriction on fattening performance, some meat quality traits and plasma leptin concentration of broilers*
}

\author{
Özlem VAROL AVCILAR ${ }^{1}$, Esin Ebru ONBAŞILAR ${ }^{2}$ \\ ${ }^{1}$ Osmaniye Korkut Ata University, Kadirli School of Applied Sciences Department of Food Technology, Kadirli, Osmaniye; ${ }^{2}$ Ankara \\ University, Faculty of Veterinary Medicine, Department of Animal Science, Ankara, Turkey.
}

\begin{abstract}
Summary: The objective of this study was to determine the effect of breeder age and early energy restriction on fattening performance, meat quality and plasma leptin concentration of broilers. A total of 504 male chicks were obtained from three different ages (27, 38 and 60 weeks) of broiler breeders. Chicks from each age of breeder groups were randomly divided into 2 diet groups (control and energy restriction). Broilers in energy restriction group were fed diet with reduced 10\% energy from day 8 to 14 . The body weight, body weight gain, feed consumption and feed to gain ratio were determined weekly during the experiment. At days 7 , 14 and 41, seven broilers from each group were bled to measure the leptin concentration. At 42 day of age, 21 broilers of each group were slaughtered. Carcass, heart, liver, gizzard, spleen and abdominal fat were weighed. pH, cooking loss, moisture, protein, fat and ash of thigh meat were determined. In conclusion, breeder age affected the initial weight, total body weight gain, feed consumption and abdominal fat, whereas energy restriction affected the $2^{\text {nd }}$ week body weight gain, total feed consumption, total feed to gain ratio, heart and liver percentages and also moisture and protein levels of thigh meat. Leptin was only affected by broiler age. Interaction between breeder age early energy restriction in diet of broilers was not seen except that the $2^{\text {nd }}$ week feed to gain ratio.
\end{abstract}

Keywords: Breeder age, broiler, early energy restriction, fattening performance, meat quality, leptin.

\section{Etçi piliçlerde damızlık yaşı ve erken dönem enerji kısıtlamasının besi performansı, bazı et kalite özellikleri ve plazma leptin konsantrasyonuna etkisi}

Özet: Bu araştırmanın amacı, etçi piliçlerde damızlık yaşı ve erken dönem enerji kısıtlamasının besi performansı, et kalitesi ve plazma leptin konsantrasyonuna etkilerini incelemektir. Toplam 504 erkek civciv üç farklı yaştaki (27, 38 ve 60 haftalık) damızlıklardan temin edilmiştir. Her damızlık yaş grubundan elde edilen civcivler rasgele iki beslenme grubuna (kontrol ve enerji kısıtlaması uygulanan grup) bölünmüştür. Enerji kısıtlaması uygulanan gruptaki etçi piliçler 8-14 günlük yaşta \%10 enerjisi azaltılmış rasyonla beslenmiştir. Deneme boyunca canlı ağırlık, canlı ağırlık artışı, yem tüketimi ve yemden yararlanma oranı haftada bir belirlenmiştir. 7, 14 ve 41. günlerde her gruptaki 7 etçi piliçten kan alınarak leptin konsantrasyonu ölçülmüştür. 42 günlük yaşta her gruptan 21 piliç kesilerek, karkas, kalp, karaciğer, taşlık, dalak ve karın yağı ağılıkları belirlenmiştir. But eti pH, pişirme kaybı, nem, protein, yağ ve kül düzeyleri tespit edilmiştir. Sonuç olarak, damızlık yaşının başlangıç canlı ağırlığı, toplam canlı ağırlık kazancı, toplam yem tüketimi ve karın yağını; enerji kısıtlamasının ikinci hafta canlı ağırlık kazancı, toplam yem tüketimi, toplam yemden yararlanma oranı, kalp ve karaciğer yüzdeleri ile but eti nem ve protein düzeylerini etkilediği belirlenmiştir. Leptin konsantrasyonu sadece etçi piliçlerin yaşından etkilenmiştir. Damızlık yaşı ile enerji kısıtlaması arasında 2. hafta yemden yararlanma oranı dışında herhangi bir etkileşim görülmemiştir.

Anahtar sözcükler: Besi performansı, damızlık yaşı, erken dönem enerji kısıtlaması, et kalitesi, etçi piliç, leptin.

\section{Introduction}

Improvements in genetics and nutrition of the broilers have led to continuing improvement in feed conversion and carcass yield. However increased growth rate has caused a greater incidence of metabolic disorders such as asides, sudden death syndrome and skeletal abnormalities and also increased fat deposition (33, 34,
39). For this purpose, quantitative and qualitative feed restriction methods such as energy restriction have been used. Leterrier et al. (22) reported that a diet with a $28 \%$ dilution of the energy, distributed in the form of finely ground particles during the first 3 week of life, induced a marked reduction in growth rate. Some studies have demonstrated that energy reduction both by feed

\footnotetext{
* This study was summarized from the PhD thesis and supported by The Scientific and Technological Research Council of Turkey (1110690).
} 
deprivation and diet dilution could decrease fat content and increase protein deposition in carcass, thus resulting in improved carcass quality $(20,40)$. Feed restriction programs are strategies that can be used to alter feeding management in order to decrease to some extent feed consumption and therefore growth rate, alleviating the occurrence of metabolic disorders and improving feed efficiency (33). The response of broilers to feed restriction depends on the duration, timing and severity of feed restriction, condition of re-alimentation and also sex and strain $(12,17,21,28,33,39,41)$. Leptin, the product of the ob gene, is a $16-\mathrm{kDa}$ hormone that has been shown to play a key role in regulating feed intake and energy homeostasis in mammals (10). Adiposities produce and secrete more leptin as fat storage increases, signaling the brain to reduce feed consumption and increase energy expenditure (1). Unlike in mammals, chicken leptin is expressed in the liver and yolk sac besides adipose tissue $(1,15,36)$. However, there is no information regarding the effects of breeder age and energy restriction on growth, plasma leptin concentration, carcass characteristics and meat composition of broilers. Older hens lay larger eggs that hatch into larger chicks and hatching weight of chicks are correlated with market age weight $(35,37)$. Thus, it was hypothesized that breeder age would affect the results of energy restriction of broilers. Based on this knowledge, adequate information is needed on the energy restriction of broilers obtained from different breeder ages on the growth. Therefore this study was conducted to investigate the effects early energy restriction and breeder age on growth, plasma leptin concentration, some carcass characteristics and meat composition of broilers.

\section{Materials and Methods}

Birds and management: A total of 504 male chicks were obtained from three different ages $(27,38$ and 60 weeks) of broiler breeders (ROSS 308). These flocks were housed under the same environmental and management conditions in the same farm. This study was approved by Ankara University Animal Care and Use Committee (2011-112-428). Chicks from each age of breeder groups were randomly divided into 2 diet groups [control (no energy restriction) and energy restriction]. There were 7 replicate pens in breeder age and diet groups. Chicks of each group were placed in separate floor pen measured as 170X94X90 cm, widthXlengthXheight, respectively. Each pen had wood

Table 1. Composition of the diets $(\mathrm{g} / \mathrm{kg})$.

Tablo 1. Rasyonların bileşimi (g/kg).

\begin{tabular}{|c|c|c|c|}
\hline & $\begin{array}{l}\text { Starter diet } \\
(0-21 \text { day })\end{array}$ & $\begin{array}{c}\text { Energy restriction diet } \\
(8-14 \text { day })\end{array}$ & $\begin{array}{l}\text { Finisher diet } \\
\text { (22-42 day) }\end{array}$ \\
\hline Maize & 415.0 & 449.2 & 46.45 \\
\hline Wheat & 100.0 & 100.0 & 10.00 \\
\hline Soya bean meal & 243.5 & 330.0 & 17.40 \\
\hline Full fat soya & 172.0 & 0 & 17.20 \\
\hline Sun flower seed meal & 0 & 62.3 & 0 \\
\hline Poultry rendering meal & 25.0 & 25.0 & 2.50 \\
\hline Vegetable oil & 11.0 & 0 & 3.30 \\
\hline Limestone & 10.0 & 10.0 & 1.00 \\
\hline Dicalcium phosphate & 12.0 & 12.0 & 1.20 \\
\hline Salt & 2.0 & 2.0 & 0.20 \\
\hline Sodium bicarbonate & 1.0 & 1.0 & 0.10 \\
\hline Methionine & 3.0 & 3.0 & 0.25 \\
\hline Lysine & 2.0 & 2.0 & 0.10 \\
\hline Threonine & 0.5 & 0.5 & 0.05 \\
\hline Vitamin-mineral premix ${ }^{1}$ & 2.5 & 2.5 & 0.25 \\
\hline Anticoccidial $^{2}$ & 0.5 & 0.5 & 0 \\
\hline \multicolumn{4}{|c|}{ Composition (analyzed) } \\
\hline Crude protein $(\%)$ & 23.10 & 23.04 & 20.10 \\
\hline ME. kcal $/ \mathrm{kg}$ & 3010 & 2707 & 3176 \\
\hline
\end{tabular}

1: Supplied the following per kg of diet: $15000 \mathrm{IU}$ vitamin A, $2400 \mathrm{IU}$ vitamin $\mathrm{D}_{3}, 30 \mathrm{mg}$ vitamin E, $2.5 \mathrm{mg}$ vitamin $\mathrm{K}_{3}, 2.5 \mathrm{mg}$ vitamin $\mathrm{B}_{1}, 6 \mathrm{mg}$ vitamin $\mathrm{B}_{2}, 4 \mathrm{mg}$ vitamin $\mathrm{B}_{6}, 20 \mu \mathrm{g}$ vitamin $\mathrm{B}_{12}, 25 \mathrm{mg}$ niacin, $8 \mathrm{mg}$ calcium-D-panthotenate, $1 \mathrm{mg}$ folic acid, 50 mg vitamin C, $50 \mu \mathrm{g}$ D-biotin, $80 \mathrm{mg} \mathrm{Mn}, 60 \mathrm{mg} \mathrm{Zn}, 60 \mathrm{mg} \mathrm{Fe}, 5 \mathrm{mg} \mathrm{Cu}, 1 \mathrm{mg} \mathrm{I}, 0.5 \mathrm{mg} \mathrm{Co}, 0.15 \mathrm{mg}$ Se.

2: Maksiban G160 (\%50 Nicarbazin + \%50 Naracin).

1: kg rasyonda sağladıkları katkı: $15000 \mathrm{IU}$ vitamin $\mathrm{A}, 2400 \mathrm{IU}$ vitamin $\mathrm{D}_{3}, 30 \mathrm{mg}$ vitamin $\mathrm{E}, 2.5 \mathrm{mg}$ vitamin $\mathrm{K}_{3}, 2.5 \mathrm{mg}$ vitamin $\mathrm{B}_{1}, 6 \mathrm{mg}$ vitamin $\mathrm{B}_{2}, 4 \mathrm{mg}$ vitamin $\mathrm{B}_{6}, 20 \mu \mathrm{g}$ vitamin $\mathrm{B}_{12}, 25 \mathrm{mg}$ niasin, $8 \mathrm{mg}$ kalsiyum-D-pantotenat, $1 \mathrm{mg}$ folik asit, $50 \mathrm{mg}$ vitamin C, $50 \mu \mathrm{g}$ D-biotin, $80 \mathrm{mg} \mathrm{Mn}, 60 \mathrm{mg} \mathrm{Zn}, 60 \mathrm{mg} \mathrm{Fe}, 5 \mathrm{mg} \mathrm{Cu}, 1 \mathrm{mg} \mathrm{I}, 0.5 \mathrm{mg}$ Co, $0.15 \mathrm{mg} \mathrm{Se}$.

2: Maksiban G160 (\%50 Nikarbazin + \%50 Narasin). 
shavings litter. During the first week each pen was equipped with one chick drinker and one chick feeder and the other weeks each pen was equipped with two nipples and one hanging suspended feeder. Feed and water were provided ad libitum during the experiment. The poultry house temperature was maintained at $33^{\circ} \mathrm{C}$ for the first $5 \mathrm{~d}$ and then gradually reduced to $21^{\circ} \mathrm{C}$. Continuous lighting was maintained throughout the experiment.

Experimental diets: All the birds were fed on a broiler starter diet for the first week $(3010 \mathrm{kcal} / \mathrm{kg} \mathrm{ME}$ and $23.10 \% \mathrm{CP}$ ). From day 8 to 14 , chicks that each breeder age groups were divided two diet groups and one of them were fed the same starter diet and the other were fed diet with reduced $10 \%$ energy $(2707 \mathrm{kcal} / \mathrm{kg} \mathrm{ME}$ and $23.04 \% \mathrm{CP}$ ). Then all broilers were provided with the same starter diet as the first week from d 15 to 21 and finisher diet from d 22 to $42(3176 \mathrm{kcal} / \mathrm{kg} \mathrm{ME}$ and $20.10 \%$ CP, Table 1).

Traits measured: The body weight, body weight gain and feed consumption were measured weekly by pen and feed conversion ratio was calculated as the feed to gain ratio. At days 7, 14 and 41, seven broilers from each group were randomly selected and bled from the brachial vein. Blood samples were taken in tubes contained EDTA. Plasma leptin concentration was determined using a commercial Gallus Leptin kit (E90084Ga, USCN) in the microplate reader (Chro-Mate, 4300).

At 42 day of age, 21 broilers of each group were randomly selected for processing. After final weighing feed was removed $8 \mathrm{~h}$ prior to slaughter. Broilers were slaughtered in a commercial slaughterhouse. Carcass, heart, liver, gizzard, spleen and abdominal fat were weighed. These weights were expressed as percentage of slaughter weight. The carcasses were stored at $4{ }^{\circ} \mathrm{C}$ for 20 $\mathrm{h}$ by hanging. Cold carcass weights were recorded and were expressed as percentage of slaughter weight as cold carcass yield. $\mathrm{pH}$ of thigh meat at $24 \mathrm{~h}$ after the slaughter was measured with pH-meter (pH-2004, J.P. Selecta, Barcelona, Spain). To determine cooking losses, samples of thigh meat were weighed, held in plastic bags, and immersed in a water bath $\left(80^{\circ} \mathrm{C}\right)$ for $20 \mathrm{~min}$. Then, bags were cooled in water for $20 \mathrm{~min}$ and samples were blotted dry with paper towels and weighed. Cooking loss $(\mathrm{CL})$ is the ratio $(\times 100)$ of the difference in weight between the cooked and raw muscle relative to the weight of the raw muscle (14). Thigh meat was also used for analyses of moisture, protein, fat and ash according to the AOAC (2). Results were expressed as percentage over fresh matter basis.

Statistical analyses: Statistical analyses were performed using the software package SPSS for Windows (SPSS Inc., Chicago, IL). Data were tested for distribution normality and homogeneity of variance. Data set showed normality and a two-way ANOVA was used to determine the differences between breeder age and diet groups as well as their interactions with respect to the studied parameters except that the leptin level. Plasma leptin level was analyzed by there-way ANOVA. Comparisons among means were made by Duncan's multiple range test ( 8 ). A value of $\mathrm{P}<0.05$ was considered statistically significant.

\section{Results}

Effects of breeder age and early energy restriction on chick weight, body weight gain, feed consumption and feed to gain ratio were summarized in Table 2, 3 and

Table 2. Effects of breeder age and early energy restriction on chick weight and body weight gain of broilers.

Tablo 2. Etçi piliçlerde damızlık yaşı ve enerji kısıtlamasının civciv ağırlığı ve canlı ağırlık artışı üzerine etkileri

\begin{tabular}{|c|c|c|c|c|c|c|c|c|c|}
\hline \multirow{2}{*}{$\begin{array}{c}\text { Breeder } \\
\text { age } \\
\text { (week) }\end{array}$} & \multirow{2}{*}{$\begin{array}{c}\text { Early } \\
\text { energy } \\
\text { restriction }\end{array}$} & \multirow{2}{*}{$\begin{array}{c}\text { Initial } \\
\text { weight (g) }\end{array}$} & \multicolumn{7}{|c|}{ Body weight gain (g) } \\
\hline & & & $1^{\text {st }}$ week & $2^{\text {nd }}$ week & $3^{\text {rd }}$ week & $4^{\text {th }}$ week & $5^{\text {th }}$ week & $6^{\text {th }}$ week & $\begin{array}{l}\text { Weeks from } \\
1 \text { to } 6\end{array}$ \\
\hline 27 & & $36.8 \pm 0.3^{\mathrm{c}}$ & $94 \pm 3^{c}$ & $218 \pm 7^{\mathrm{b}}$ & $399 \pm 11^{b}$ & $649 \pm 16^{\mathrm{b}}$ & $625 \pm 21^{b}$ & $676 \pm 23$ & $2660 \pm 35^{\mathrm{b}}$ \\
\hline 38 & & $41.7 \pm 0.3^{\mathrm{b}}$ & $128 \pm 3^{\mathrm{b}}$ & $265 \pm 7^{\mathrm{a}}$ & $438 \pm 11^{\mathrm{a}}$ & $705 \pm 16^{\mathrm{a}}$ & $676 \pm 21^{\mathrm{ab}}$ & $667 \pm 23$ & $2878 \pm 35^{\mathrm{a}}$ \\
\hline \multirow[t]{3}{*}{60} & & $48.2 \pm 0.3^{\mathrm{a}}$ & $144 \pm 3^{\mathrm{a}}$ & $282 \pm 7^{\mathrm{a}}$ & $468 \pm 11^{\mathrm{a}}$ & $724 \pm 16^{\mathrm{a}}$ & $706 \pm 21^{\mathrm{a}}$ & $618 \pm 23$ & $2942 \pm 35^{\mathrm{a}}$ \\
\hline & - & $42.2 \pm 0.3$ & $121 \pm 2$ & $267 \pm 5$ & $426 \pm 9$ & $684 \pm 13$ & $656 \pm 17$ & $669 \pm 19$ & $2824 \pm 28$ \\
\hline & + & $42.2 \pm 0.3$ & $123 \pm 2$ & $242 \pm 5$ & $443 \pm 9$ & $701 \pm 13$ & $682 \pm 17$ & $638 \pm 19$ & $2829 \pm 28$ \\
\hline \multicolumn{2}{|c|}{ Expected mean $(\mu)$} & $42.2 \pm 0.2$ & $122 \pm 2$ & $255 \pm 4$ & $435 \pm 6$ & $692 \pm 10$ & $669 \pm 12$ & $654 \pm 13$ & $2826 \pm 20$ \\
\hline \multicolumn{2}{|c|}{ Breeder age } & $* * *$ & $* * *$ & $* * *$ & $* * *$ & $* *$ & $*$ & NS & $* * *$ \\
\hline \multicolumn{2}{|c|}{$\begin{array}{l}\text { Early energy } \\
\text { restriction }\end{array}$} & NS & NS & $* *$ & NS & NS & NS & NS & NS \\
\hline \multicolumn{2}{|c|}{$\begin{array}{l}\text { Breeder age X Early } \\
\text { energy restriction }\end{array}$} & NS & NS & NS & NS & NS & NS & NS & NS \\
\hline
\end{tabular}

$\mathrm{n}=7$, -: No energy restriction, $+: 10 \%$ energy restriction from day 8 to 14 . Values are estimated mean \pm SEM, ${ }^{\mathrm{a}, \mathrm{b}, \mathrm{c}}{ }^{\mathrm{a}}$ : Means within columns with different letters are significantly different $(\mathrm{P}<0.05)$. NS: non significant, $*$ : $\mathrm{P}<0.05, * *: \mathrm{P}<0.01, \quad * * *$ : $\mathrm{P}<0.001$. $\mathrm{n}=7$, -: Enerji kısıtlaması yapılmayan, $+: 8$ ile 14. günlerde $\% 10$ enerji kısıtlaması yapılan. Değerler tahmin edilen ortalama $\pm \mathrm{SH}$

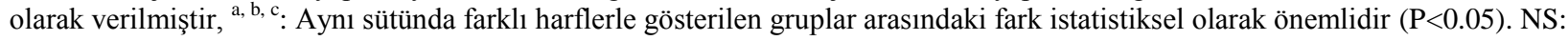
önemli değil, *: $\mathrm{P}<0.05, * *$ : $\mathrm{P}<0.01, \quad * * *$ : $\mathrm{P}<0.001$. 
4. Breeder age affected the initial weight, total body weight gain and feed consumption. There was no significant difference in feed to gain ratio among different breeder age groups. Energy restriction affected the $2^{\text {nd }}$ week body weight gain, total feed consumption, total feed to gain ratio. Hot carcass yield, percentages of heart, liver, spleen and gizzard and also thigh meat composition and cooking loss were not affected by the breeder age groups (Table 5 and 6). Abdominal fat of broilers obtained from 27 week old breeders was significantly lower $(\mathrm{P}<0.05)$ than that of broilers obtained from 38 week old breeder. Early energy restriction affected heart and liver percentages and also protein and moisture levels of thigh meat. Leptin was only affected by broiler age $(\mathrm{P}<0.001)$ (Table 7$)$.

\section{Discussion and Conclusion}

Initial chick weight obtained from 27 week old breeder age was lower $(\mathrm{P}<0.001)$ than that obtained from older breeders. It is expected because the smaller eggs produced young broiler breeders and there was positive correlation between egg weight and hatched chick weight (37). Heavy chicks has got high nutrition reserve and show high live rate (26). Chick weight accepted as egg weight percentage in hens and this rate is $62-76 \%$ in chickens (37). Breeder age affected the body weight gain of broilers until $6^{\text {th }}$ week. At these weeks broilers obtained from 27 week old breeders were lower body weight gain than broilers obtained from 60 week old breeders. Total body weight gain from $1^{\text {st }}$ to $6^{\text {th }}$ week was also lower in broilers obtained from 27 week old breeders than that obtained elders $(\mathrm{P}<0.001)$. However initial body

Table 3. Effects of breeder age and early energy restriction on feed consumption of broilers.

Tablo 3. Etçi piliçlerde damızlık yaşı ve enerji kısıtlamasının yem tüketimine etkisi

\begin{tabular}{|c|c|c|c|c|c|c|c|c|}
\hline \multirow{2}{*}{$\begin{array}{l}\text { Breeder } \\
\text { age } \\
\text { (week) }\end{array}$} & \multirow{2}{*}{$\begin{array}{c}\text { Early } \\
\text { energy } \\
\text { restriction }\end{array}$} & \multicolumn{7}{|c|}{ Feed consumption (g/broiler) } \\
\hline & & $1^{\text {st }}$ week & $2^{\text {nd }}$ week & $3^{\text {rd }}$ week & $4^{\text {th }}$ week & $5^{\text {th }}$ week & $6^{\text {th }}$ week & $\begin{array}{l}\text { Weeks from } \\
1 \text { to } 6\end{array}$ \\
\hline 27 & & $111 \pm 4^{\mathrm{c}}$ & $401 \pm 18^{\mathrm{b}}$ & $571 \pm 21^{b}$ & $907 \pm 22^{b}$ & $1007 \pm 20^{\mathrm{b}}$ & $1166 \pm 23^{\mathrm{a}}$ & $4162 \pm 57^{\mathrm{b}}$ \\
\hline 38 & & $143 \pm 4^{\mathrm{b}}$ & $488 \pm 18^{\mathrm{a}}$ & $642 \pm 21^{\mathrm{a}}$ & $1037 \pm 22^{\mathrm{a}}$ & $1070 \pm 20^{\mathrm{a}}$ & $1123 \pm 23^{\mathrm{ab}}$ & $4503 \pm 57^{\mathrm{a}}$ \\
\hline \multirow[t]{3}{*}{60} & & $166 \pm 4^{\mathrm{a}}$ & $529 \pm 18^{\mathrm{a}}$ & $664 \pm 21^{\mathrm{a}}$ & $1040 \pm 22^{\mathrm{a}}$ & $1081 \pm 20^{\mathrm{a}}$ & $1068 \pm 23^{\mathrm{b}}$ & $4548 \pm 57^{\mathrm{a}}$ \\
\hline & - & $141 \pm 4$ & $403 \pm 15$ & $614 \pm 17$ & $990 \pm 18$ & $1017 \pm 17$ & $1142 \pm 19$ & $4305 \pm 46$ \\
\hline & + & $139 \pm 4$ & $543 \pm 15$ & $638 \pm 17$ & $999 \pm 18$ & $1088 \pm 17$ & $1096 \pm 19$ & $4503 \pm 46$ \\
\hline \multicolumn{2}{|c|}{ Expected mean $(\mu)$} & $140 \pm 3$ & $473 \pm 11$ & $626 \pm 12$ & $995 \pm 13$ & $1053 \pm 12$ & $1119 \pm 13$ & $4404 \pm 33$ \\
\hline \multicolumn{2}{|c|}{ Breeder age } & $* * *$ & $* * *$ & $* *$ & $* * *$ & $*$ & $*$ & $* * *$ \\
\hline \multicolumn{2}{|c|}{ Early energy restriction } & NS & $* * *$ & NS & NS & $* *$ & NS & $* *$ \\
\hline \multicolumn{2}{|c|}{$\begin{array}{l}\text { Breeder age } X \text { Early } \\
\text { energy restriction }\end{array}$} & NS & NS & NS & NS & NS & NS & NS \\
\hline
\end{tabular}

$\mathrm{n}=7$, -: No energy restriction, +: $10 \%$ energy restriction from day 8 to 14 . Values are estimated mean \pm SEM, a, b,c: Means within columns with different letters are significantly different $(\mathrm{P}<0.05)$. NS: non significant, *: $\mathrm{P}<0.05, * *: \mathrm{P}<0.01, * * *: \mathrm{P}<0.001$.

$\mathrm{n}=7$, -: Enerji kısıtlaması yapılmayan, $+: 8$ ile 14. günlerde $\% 10$ enerji kisitlaması yapılan. Değerler tahmin edilen ortalama $\pm \mathrm{SH}$ olarak verilmiştir, ${ }^{\mathrm{a}, \mathrm{b}, \mathrm{c}}$ : Aynı sütünda farklı harflerle gösterilen gruplar arasındaki fark istatistiksel olarak önemlidir $(\mathrm{P}<0.05)$. NS: önemli değil, *: $\mathrm{P}<0.05, \quad * *: \mathrm{P}<0.01, \quad * * *: \mathrm{P}<0.001$.

Table 4. Effects of breeder age and early energy restriction on feed to gain ratio of broilers.

Tablo 4. Etçi piliçlerde damızlık yaşı ve enerji kısıtlamasının yemden yararlanma oranına etkisi.

\begin{tabular}{|c|c|c|c|c|c|c|c|c|}
\hline \multirow{2}{*}{$\begin{array}{c}\text { Breeder } \\
\text { age } \\
\text { (week) }\end{array}$} & \multirow{2}{*}{$\begin{array}{c}\text { Early } \\
\text { energy } \\
\text { restriction }\end{array}$} & \multicolumn{7}{|c|}{ Feed to gain ratio $(\mathrm{g} / \mathrm{g})$} \\
\hline & & $1^{\text {st }}$ week & $2^{\text {nd }}$ week & $3^{\text {rd }}$ week & $4^{\text {th }}$ week & $5^{\text {th }}$ week & $6^{\text {th }}$ week & $\begin{array}{c}\text { Weeks from } \\
1 \text { to } 6\end{array}$ \\
\hline 27 & & $1.18 \pm 0.02$ & $1.91 \pm 0.07$ & $1.43 \pm 0.04$ & $1.41 \pm 0.03$ & $1.64 \pm 0.05$ & $1.75 \pm 0.05$ & $1.57 \pm 0.02$ \\
\hline 38 & & $1.12 \pm 0.02$ & $1.86 \pm 0.07$ & $1.47 \pm 0.04$ & $1.47 \pm 0.03$ & $1.59 \pm 0.05$ & $1.71 \pm 0.05$ & $1.57 \pm 0.02$ \\
\hline \multirow[t]{3}{*}{60} & & $1.15 \pm 0.02$ & $1.89 \pm 0.07$ & $1.43 \pm 0.04$ & $1.44 \pm 0.03$ & $1.54 \pm 0.05$ & $1.74 \pm 0.05$ & $1.55 \pm 0.02$ \\
\hline & - & $1.16 \pm 0.02$ & $1.51 \pm 0.06$ & $1.45 \pm 0.04$ & $1.45 \pm 0.03$ & $1.56 \pm 0.04$ & $1.73 \pm 0.04$ & $1.52 \pm 0.01$ \\
\hline & + & $1.14 \pm 0.02$ & $2.26 \pm 0.06$ & $1.44 \pm 0.04$ & $1.43 \pm 0.03$ & $1.62 \pm 0.04$ & $1.73 \pm 0.04$ & $1.60 \pm 0.01$ \\
\hline \multicolumn{2}{|c|}{ Expected mean $(\mu)$} & $1.15 \pm 0.01$ & $1.88 \pm 0.04$ & $1.44 \pm 0.03$ & $1.44 \pm 0.02$ & $1.59 \pm 0.03$ & $1.73 \pm 0.03$ & $1.56 \pm 0.01$ \\
\hline \multicolumn{2}{|c|}{ Breeder age } & NS & NS & NS & NS & NS & NS & NS \\
\hline \multicolumn{2}{|c|}{ Early energy restriction } & NS & $* * *$ & NS & NS & NS & NS & $* *$ \\
\hline \multicolumn{2}{|c|}{ Breeder age X Early } & NS & $*$ & NS & NS & NS & NS & NS \\
\hline
\end{tabular}

energy restriction

$\mathrm{n}=7,-$ : No energy restriction, $+: 10 \%$ energy restriction from day 8 to 14 . Values are estimated mean \pm SEM, NS: non significant, *: $\mathrm{P}<0.05, * *: \mathrm{P}<0.01, * * *: \mathrm{P}<0.001$.

$\mathrm{n}=7$, -: Enerji kısıtlaması yapılmayan, $+: 8$ ile 14. günlerde $\% 10$ enerji kısıtlaması yapılan. Değerler tahmin edilen ortalama $\pm \mathrm{SH}$ olarak verilmiştir, NS: önemli değil, *: $\mathrm{P}<0.05, \quad * *: \mathrm{P}<0.01$, ***: $\mathrm{P}<0.001$. 
Table 5. Effects of breeder age and early energy restriction on hot carcass yield and percentages of some organs of broilers. Tablo 5. Etçi piliçlerde damızlık yaşı ve enerji kısıtlamasının sıcak karkas randımanı ve bazı organ yüzdelerine etkisi.

\begin{tabular}{cccccccc}
\hline $\begin{array}{c}\text { Breeder age } \\
\text { (week) }\end{array}$ & $\begin{array}{c}\text { Early energy } \\
\text { restriction }\end{array}$ & $\begin{array}{c}\text { Hot carcass } \\
\text { yield } \\
(\% \mathrm{SW})\end{array}$ & $\begin{array}{c}\text { Heart } \\
(\% \mathrm{SW})\end{array}$ & $\begin{array}{c}\text { Liver } \\
(\% \mathrm{SW})\end{array}$ & $\begin{array}{c}\text { Spleen } \\
(\% \mathrm{SW})\end{array}$ & $\begin{array}{c}\text { Gizard } \\
(\% \mathrm{SW})\end{array}$ & $\begin{array}{c}\text { Abdominal } \\
\text { fat }(\% \mathrm{SW})\end{array}$ \\
\hline 27 & & $76.7 \pm 0.4$ & $0.57 \pm 0.01$ & $1.95 \pm 0.06$ & $0.105 \pm 0.003$ & $1.11 \pm 0.03$ & $1.25 \pm 0.04^{\mathrm{b}}$ \\
38 & & $76.0 \pm 0.4$ & $0.57 \pm 0.01$ & $1.91 \pm 0.06$ & $0.103 \pm 0.003$ & $1.09 \pm 0.03$ & $1.40 \pm 0.04^{\mathrm{a}}$ \\
60 & - & $76.0 \pm 0.4$ & $0.54 \pm 0.01$ & $1.79 \pm 0.06$ & $0.106 \pm 0.003$ & $1.16 \pm 0.03$ & $1.34 \pm 0.04^{\mathrm{ab}}$ \\
& + & $76.4 \pm 0.3$ & $0.57 \pm 0.01$ & $1.95 \pm 0.05$ & $0.105 \pm 0.002$ & $1.12 \pm 0.02$ & $1.36 \pm 0.03$ \\
Expected mean $(\mu)$ & $76.1 \pm 0.3$ & $0.54 \pm 0.01$ & $1.81 \pm 0.05$ & $0.102 \pm 0.002$ & $1.12 \pm 0.02$ & $1.30 \pm 0.03$ \\
Breeder age & $76.2 \pm 0.2$ & $0.56 \pm 0.01$ & $1.88 \pm 0.03$ & $0.105 \pm 0.002$ & $1.118 \pm 0.02$ & $1.33 \pm 0.02$ \\
Early energy restriction & $\mathrm{NS}$ & $\mathrm{NS}$ & $\mathrm{NS}$ & $\mathrm{NS}$ & $\mathrm{NS}$ & $*$ \\
Breeder age X Early energy restriction & $\mathrm{NS}$ & $* *$ & $*$ & $\mathrm{NS}$ & $\mathrm{NS}$ & $\mathrm{NS}$ \\
\hline
\end{tabular}

$\mathrm{n}=21,-$ : No energy restriction, $+: 10 \%$ energy restriction from day 8 to $14, \mathrm{SW}$ : Slaughter weight. Values are estimated mean \pm SEM, ${ }^{\mathrm{a}, \mathrm{b}}$ : Means within columns with different letters are significantly different $(\mathrm{P}<0.05)$. NS: non significant, *: $\mathrm{P}<0.05, * *: \mathrm{P}<0.01$. $\mathrm{n}=21$, -: Enerji kısıtlaması yapılmayan, $+: 8$ ile 14. günlerde $\% 10$ enerji kısıtlaması yapılan. Değerler tahmin edilen ortalama $\pm \mathrm{SH}$ olarak verilmiştir, ${ }^{\mathrm{a}, \mathrm{b}}$ : Aynı sütünda farklı harflerle gösterilen gruplar arasındaki fark istatistiksel olarak önemlidir $(\mathrm{P}<0.05)$. NS: önemli değil, *: $\mathrm{P}<0.05, * *: \mathrm{P}<0.01$.

Table 6. Effects of breeder age and early energy restriction on thigh meat composition, $\mathrm{pH}$ and cooking loss.

Tablo 6. Etçi piliçlerde damızlık yaşı ve enerji kısıtlamasının but eti bileşimi, pH ve pişirme kaybına etkisi.

\begin{tabular}{|c|c|c|c|c|c|c|c|}
\hline $\begin{array}{l}\text { Breeder age } \\
\text { (week) }\end{array}$ & $\begin{array}{c}\text { Early energy } \\
\text { restriction }\end{array}$ & $\begin{array}{c}\text { Moisture } \\
\text { (\% on fresh } \\
\text { matter) }\end{array}$ & $\begin{array}{c}\text { Ash } \\
\text { (\% on fresh } \\
\text { matter) }\end{array}$ & $\begin{array}{c}\text { Protein } \\
\text { (\% on fresh } \\
\text { matter) }\end{array}$ & $\begin{array}{c}\text { Fat } \\
\text { (\% on fresh } \\
\text { matter) }\end{array}$ & $\mathrm{pH}$ & $\begin{array}{l}\text { Cooking } \\
\text { loss }(\%)\end{array}$ \\
\hline 27 & & $74.79 \pm 0.31$ & $1.07 \pm 0.01$ & $20.41 \pm 0.22$ & $3.73 \pm 0.14$ & $6.16 \pm 0.03$ & $23.51 \pm 0.99$ \\
\hline 38 & & $75.18 \pm 0.31$ & $1.08 \pm 0.01$ & $19.85 \pm 0.22$ & $3.74 \pm 0.14$ & $6.13 \pm 0.03$ & $21.47 \pm 0.99$ \\
\hline \multirow[t]{3}{*}{60} & & $75.16 \pm 0.31$ & $1.11 \pm 0.01$ & $19.88 \pm 0.22$ & $3.74 \pm 0.14$ & $6.11 \pm 0.03$ & $22.37 \pm 0.99$ \\
\hline & - & $74.69 \pm 0.25$ & $1.08 \pm 0.01$ & $20.32 \pm 0.18$ & $3.78 \pm 0.12$ & $6.12 \pm 0.02$ & $22.60 \pm 0.81$ \\
\hline & + & $75.41 \pm 0.25$ & $1.10 \pm 0.01$ & $19.78 \pm 0.18$ & $3.69 \pm 0.12$ & $6.15 \pm 0.02$ & $22.30 \pm 0.81$ \\
\hline Expected mean & & $75.05 \pm 0.18$ & $1.09 \pm 0.01$ & $20.11 \pm 0.16$ & $3.73 \pm 0.08$ & $6.14 \pm 0.02$ & $22.45 \pm 0.57$ \\
\hline \multicolumn{2}{|c|}{ Breeder age } & NS & NS & NS & NS & NS & NS \\
\hline \multicolumn{2}{|c|}{ Early energy restriction } & $*$ & NS & $*$ & NS & NS & NS \\
\hline \multicolumn{2}{|c|}{ Breeder age $X$ Early energy restriction } & NS & NS & NS & NS & NS & NS \\
\hline
\end{tabular}

$\mathrm{n}=7$, -: No energy restriction, $+: 10 \%$ energy restriction from day 8 to 14 . Values are estimated mean \pm SEM, NS: non significant, *: $\mathrm{P}<0.05$.

n=7, -: Enerji kısıtlaması yapılmayan, +: 8 ile 14. günlerde $\% 10$ enerji kısıtlaması yapılan. Değerler tahmin edilen ortalama \pm SH olarak verilmiștir, NS: önemli değil, *: $\mathrm{P}<0.05$.

Table 7. Effects of breeder age and early energy restriction on plasma leptin concentrations (ng/ml) of broilers in different period. Tablo 7. Etçi piliçlerde damızlık yaşı ve enerji kısıtlamasının farklı dönemlerde plazma leptin (ng/ml) konsantrasyonlarına etkisi.

\begin{tabular}{|c|c|c|c|}
\hline Period (day) & Breeder age (week) & Early energy reduction & Plasma leptin concentration \\
\hline 7 & & & $1.47 \pm 0.06^{\mathrm{a}}$ \\
\hline 14 & & & $1.40 \pm 0.06^{\mathrm{a}}$ \\
\hline \multirow[t]{6}{*}{41} & & & $0.52 \pm 0.06^{\mathrm{b}}$ \\
\hline & 27 & & $1.14 \pm 0.06$ \\
\hline & 38 & & $1.08 \pm 0.06$ \\
\hline & 60 & & $1.17 \pm 0.06$ \\
\hline & & - & $1.15 \pm 0.05$ \\
\hline & & + & $1.11 \pm 0.05$ \\
\hline \multicolumn{2}{|c|}{ Expected mean $(\mu)$} & & $1.13 \pm 0.04$ \\
\hline \multicolumn{2}{|l|}{ Period } & & $* * *$ \\
\hline \multicolumn{2}{|l|}{ Breeder age } & & NS \\
\hline \multicolumn{2}{|c|}{ Early energy reduction } & & NS \\
\hline \multicolumn{2}{|c|}{ PeriodXBreeder ageXEarly energy reduction } & & NS \\
\hline
\end{tabular}

$\mathrm{n}=7$, -: No energy restriction, +: $10 \%$ energy restriction from day 8 to 14 . Values are estimated mean \pm SEM, ${ }^{\text {a,b }}$ Means within columns with different letters are significantly different $(\mathrm{P}<0.05)$. NS: non significant, $* * *$ : $\mathrm{P}<0.001$.

$\mathrm{n}=7$, -: Enerji kısıtlaması yapılmayan, $+: 8$ ile 14. günlerde $\% 10$ enerji kısıtlaması yapılan. Değerler tahmin edilen ortalama $\pm \mathrm{SH}$ olarak verilmiştir, ${ }^{\mathrm{a}, \mathrm{b}}{ }^{\mathrm{S}}$ : Aynı sütünda farklı harflerle gösterilen gruplar arasındaki fark istatistiksel olarak önemlidir $(\mathrm{P}<0.05)$. NS: önemli değil, $* * *: \mathrm{P}<0.001$. 
weights and first week body weight gains were significantly different in broilers obtained from 38 and 60 weeks old breeders, after the first week body weight gains were statistically similar in these groups. In our study, $10 \%$ energy restriction in the diets was done from day 8 to 14 . Therefore in diet groups, body weight gain was only affected in the second week. In this week broilers in energy restriction group gained significantly less $(\mathrm{P}<0.01)$ body weight than the broilers in control group. After this week body weight gains in diet groups were statistically similar. The results of our study demonstrate that reducing $10 \%$ dietary energy from day 8 to 14 of age resulted in reduced weight gain this week; however full recovery in growth of broilers previously fed energy reduced diets can be achieved. Yang et al. (38) reported similar results that restriction of $10 \%$ of energy had no influence on weight gain. However, Fontana et al. (11) showed that male chicks provided with a lower energy diet for 7 day from 4 day of age were unable to normalize weight gain and had significantly lower body weight at 49 day. This difference among studies might be due to the severity and timing of restriction method.

First week feed consumption of broilers among breeder age groups were statistically different $(\mathrm{P}<0.001)$. In this week chicks obtained from young breeders consumed little feed than chicks obtained from old breeders. After the first week chicks obtained from 38 and 60 weeks old breeders consumed statistically similar feed. However chicks obtained from 27 week old breeders consumed less than the others until the $6^{\text {th }}$ week. These broilers in the $6^{\text {th }}$ week consumed more feed than that of the older breeders. However Onbaşılar et al. (27) showed that feed consumptions of broilers among different breeder ages were not statistically different. This difference between studies might be due to the using different breeder age groups. Broilers in early energy restriction group from the $2^{\text {nd }}$ week to $5^{\text {th }}$ week consumed more feed than in control group. But only $2^{\text {nd }}$ and $5^{\text {th }}$ weeks (respectively, $\mathrm{P}<0.001$ and $\mathrm{P}<0.01$ ) and also total weeks were statistically significant $(\mathrm{P}<0.01)$. Similarly, Bregendahl et al. (5) and Nawaz et al. (25) reported that increase feed consumption in broilers fed diets having decreased energy. Griffiths et al. (13) reported that feed consumption of broilers decreased as dietary energy increased. Similarly, in our study showed that early energy restriction was increased the feed consumption of broilers.

There was no significant difference in feed to gain ratio among different breeder age groups. Feed to gain ratio of broilers in energy restriction group was statistically higher $(\mathrm{P}<0.001)$ than that of the broilers in control group at the $2^{\text {nd }}$ week and also weeks from 1 to 6 $(\mathrm{P}<0.01)$. The increase in feed to gain ratio was associated with increase feed consumption and decrease weight gain. Similarly, Leterrier et al. (22) reported that broilers fed low energy diet for 3 week had low body weight. During the first $42 \mathrm{~d}$ of life, broilers fed low energy showed a $10.8 \%$ increase in feed consumption. Higher feed to gain ratio was observed among broilers fed low energy in their study.

Hot carcass yield, percentages of heart, liver, spleen and gizzard were not affected by the breeder age groups. Abdominal fat was affected by breeder age groups. Abdominal fat of broilers obtained from 27 week old breeders was significantly lower $(\mathrm{P}<0.05)$ than that of broilers obtained from 38 week old breeder. Similarly, Onbaşılar et al. (27) reported that abdominal fat ratio of broilers obtained from young breeder was lower than that of broilers obtained from old breeders. Meat quality traits vary with growth rate (19). Meat from broilers with higher growth rate had relatively large fibres and $\mathrm{pH} 24$, dark colour and low thawing loss (4). Composition, $\mathrm{pH}$ and cooking loss of thigh meat were not affected by the breeder age groups.

One of the most controversial aspects of early feed restriction methods has been the lack of a consistent effect on abdominal fat. A reduction in abdominal fat without any concomitant reduction in body weight was reported some authors $(16,30,31,32)$ Other authors $(6$, 24) have reported reduction in abdominal fat due to early feed restriction but with a small reduction in final body weight. Yang et al. (38) reported that broilers receiving energy restriction diets had lower fat deposition. Lippens et al. (23) reported that total fat content was not changed by feed restriction. Similarly, our results showed that early energy restriction didn't affect the abdominal fat. Normally muscle $\mathrm{pH}$ is about 7.0 and rapidly declines after slaughter. In this study $\mathrm{pH}$ of the thigh meat, $24 \mathrm{~h}$ after slaughter, reached an average of 6.14 for diet groups. Groups of diet had no significant affect on this variable. Diets only affected the percentages of heart and liver and also moisture and protein levels of thigh meats. Rapid growth necessitates high oxygen demand. Implementation of feed restriction program, therefore, reduces the growth rate and results in decreased tissue oxygen consumption (3). In this study reduced growth rate of broilers due to the early energy restriction in the $2^{\text {nd }}$ week decreased the heart $(\mathrm{P}<0.01)$ and liver $(\mathrm{P}<0.05)$ percentages. Similarly, some authors $(28,29)$ reported that early feed restriction decreased heart percentages. Yang et al. (38) found increased moisture of breast muscle in early energy restricted broilers and day 42 but not statistically significant. However, Zhan et al. (40) reported that increased fat and similar protein contents of breast muscle in feed restricted broilers as compared with broilers fed adlibitum at the end of restriction. In our study, moisture was higher and protein was lower in 
thigh meat of broilers fed energy restricted diet on day 42 $(\mathrm{P}<0.05)$. The difference in protein content of the muscle could be explained by protein metabolism and examined region of muscle due to the different growth time of the different body region.

We thought that because of differential growth rate, feed consumption and abdominal fat of broilers obtained from different breeder ages, plasma leptin concentrations were different. According to Dridi and Taouis (9), chickens of heavier body weights have higher leptin concentrations in the plasma than those with lower body weights. In our study, while broilers from three breeder age groups had different body weights, weight gains and abdominal fat, plasma concentrations of leptin were statistically similar. Similarly Kuo et al. (18) reported that plasma leptin concentration may not be the cause for differences in body weight and food intake between low and high body weight chickens. Liver percentages were similar in breeder age groups; therefore in broilers liver might determine the plasma leptin level primarily. However, in energy restriction groups, plasma leptin concentrations were similar but liver percentages were statistically different $(\mathrm{P}<0.05)$ in diet groups. This result was not support to previous result about leptin concentrations of broilers obtained from different breeder ages. The present experiment also showed that plasma leptin concentrations decreased $(\mathrm{P}<0.001)$ with age in broilers. This could be related to in ovo embryonic nutrition, which in the chick is mainly supported by lipids contained in the yolk. Cassy et al. (7) reported that plasma leptin concentration was higher on day 1 than on day 35 of age, but this was not statistically different. Further studies should focus on the leptin concentration to determine these differences.

In this study, we wanted to know whether the differences observed among broilers obtained from different breeder ages due to the early feed restriction. However, the results showed that there were no significant interaction between breeder age and early energy restriction except that the $2^{\text {nd }}$ week feed to gain ratio.

As a conclusion, this study has demonstrated that initial weight, total body weight gain and total feed consumption were decreased as breeder age decreases, whereas energy restriction caused to decrease total feed efficiency, heart and liver percentages and also protein level of thigh meat. Leptin concentration was decreased as broiler age increases. Breeder age didn't influence the early dietary energy restriction of broilers except that at the $2^{\text {nd }}$ week of feed to gain ratio.

\section{Acknowledgements}

We thank the Beypiliç for supplying chicks.

\section{References}

1. Ashwell CM, Czerwinski SM, Brocht DM, McMurtry JP (1999): Hormonal regulation of leptin expression in broiler chickens. Comp Phys, 276, 226-232.

2. AOAC (2000): Official Methods of Analysis. $17^{\text {th }}$ ed. Maryland, USA, AOAC International.

3. Balog JM, Anthony NB, Cooper MA, Kidd BD, Huff GR, Huff WE, Rath NC (2000): Ascites syndrome and related pathologies in feed restricted broilers raised in a hypobaric chamber. Poult Sci, 79, 318-323.

4. Berri C, Debut M, Santé-Lhoutellier C, et al (2005): Variations in chicken breast meat quality: A strong implication of struggle and muscle glycogen level at death. Br Poult Sci, 46, 572-579.

5. Bregendahl K, Sell JL, Zimmerman DR (2002): Effect of low-protein diets on growth performance and body composition of broiler chicks. Poult Sci, 81, 1156-1167.

6. Cabel MC, Waldroup PW (1990): Effect of different nutrient-restriction programs early in life on broiler performance and abdominal fat content. Poult Sci, 69, 652-660.

7. Cassy S, Picard M, Crochet S, et al (2004): Peripheral leptin effect on food intake in young chickens is influenced by age and strain. Domes Anim Endoc, 27, 51-61.

8. Dawson B, Trapp RG (2001): Basic and Clinical Biostatistics. $3^{\text {rd }}$ ed. New York, Lange Medical Books/McGrawHill Medical Publishing Division.

9. Dridi S, Taouis M (2001): Leptin expression and regulation in chicken. Br Poult Sci, 42, 50-51

10. Friedman JM, Halaas JL (1998): Leptin and regulation of body weight in mammals. Nature, 395, 763-770.

11. Fontana EA, Weaver WD, Watkins JBA, et al (1992): Effect of early feed restriction on growth, feed conversion, and mortality in broiler chickens. Poult Sci, 71, 12961305.

12. Gous RM, Cherry P (2004): Effects of body weight at, and lighting regimen and growth curve to 20 weeks on laying performance in broiler breeders. Br Poult Sci, 45, 445-452.

13. Griffiths L, Leeson S, Summers JD (1977): Fat deposition in broilers: Effect of dietary energy to protein balance and early life caloric restriction on productive performance and abdominal fat pad size. Poult Sci, 56, 638-646.

14. Honikel KO (1998): Reference methods for the assessment of physical characteristics of meat. Meat Sci, 49, 447-457.

15. Huang JX, Luo XG, Lu L, et al (2008): Effects of age and strain on yolk sac utilization and leptin levels in newly hatched broilers. Poult Sci, 87, 2647-2652.

16. Jones GPD, Farrell DJ (1992): Early life food restriction of the chicken. I. Methods of application, amino acid supplementation and the age at which restriction should commence. Br Poult Sci, 33, 579-587.

17. Khetani TL, Nkukwana TT, Chimonyo M, et al (2008): Effect of quantitative feed dilution on broiler performance. Trop Anim Health Prod, 41, 379-384.

18. Kuo AY, Cline MA, Werner E, et al (2005): Leptin effects on food and water intake in lines of chickens selected for high or low body weight. Physiol Behav, 16, 459-464. 
19. Le Bihan-Duval E, Debut M, Berri CM, et al (2008): Chicken meat quality: genetic variability and relationship with growth and muscle characteristics. Genetics, 9, 53.

20. Lee KH, Leeson S (2001): Performance of broilers fed limited quantities of feed or nutrients during seven to fourteen days of age. Poult Sci, 80, 446-454.

21. Leeson S, Summers JD (2005): Commercial Poultry Nutrition. 3rd ed. University books, Ontario, Canada. 398 pp.

22. Leterrier C, Rose N, Constantın P, Nys Y (1998): Reducing growth rate of broiler chickens with a low energy diet does not improve cortical bone quality. Brt Poult Sci, 39, 24-30.

23. Lippens M, Room G, Groote GD, et al (2000): Early and temporary quantitative food restriction of broiler chickens. 1. Effects on performance characteristics, mortality and meat quality. Brt Poult Sci, 41, 343-354.

24. Mudric S, Bozickovic P, Krivec G (1994): Performance of broiler chicks following continuous or discontinuous early feed restriction. Proceedings of the $9^{\text {th }}$ Europian Poultry Conference, Glasgow, pp. 477-478.

25. Nawaz H, Mushtaq T, Yaqoob M (2006): Effect of varying levels of energy and protein on live performance and carcass characteristics of broiler chicks. J Poult Sci, 43, 388-393.

26. O'Connor RJ (1984): The Growth and Development of Birds. John Wiley And Sons New York, USA.

27. Onbaşılar EE, Poyraz Ö, Çetin S (2008): Effects of breeder age and stocking density on performance, carcass characteristics and some stress parameters of broilers. Asian-Aust J Anim Sci, 21, 262-269.

28. Onbaşılar EE, Yalçın S, Torlak E, et al (2009): Effects of early feed restriction on live performance, carcass characteristics,meat and liver composition, some blood parameters, heterophile lymphocyte ratio, antibody production and tonic immobility duration. Trop Anim Health Prod, 41, 1513-1519.

29. Petek M (2000): The effects of Feed removal during the day on some prodution traits and blood parameters of broilers. Turk J Vet Anim Sci, 24, 447-452.

30. Plavnik I, Hurwitz S (1985): The performance of broiler chicks during and following a severe feed restriction at an early age. Poult Sci, 64, 348-355.

31. Plavnik I, Hurwitz S (1988): Early feed restriction in chicks: Effects of age, duration and sex. Poult Sci, 67, 1407-1413.
32. Plavnik I, Hurwitz S (1991): Response of broiler chickens and turkey poults to foodrestriction of varied severity during early life. Br Poult Sci, 32, 343-352.

33. Rincon MU (2000): Mild feed restriction and compensatory growth in broiler chicks. M.Sc. Thesis, Graduate School, University of Guelph, Canada.

34. Robinson FE, Classen HL, Hanson JA, et al (1992): Growth Performance, Feed Efficiency and the Incidence of Skeletal and Metabolic Disease in Full-Fed and Feed Restricted Broiler and Roaster Chickens. J App Poult Res, 1, 33-41.

35. Shanawany MM (1987): Hatching Weight in Relation to Egg Weight in Domestic Birds. World's Poult Sci J, 43, 107-115.

36. Taouis M, Chen JW, Daviaud C, et al (1998): Cloning the chicken leptin gene. Gene, 208, 239-242.

37. Wilson HR (1991): Interrelationship of egg size, chick size, posthatching growth, and hatchability. World's Poult Sci J, 47, 5-20.

38. Yang YX, Guo J, Yoon SY, et al (2009): Early energy and protein reduction: efects on growth, blood profiles and expression of genes related to protein and fat metabolism in broilers. Br Poult Sci, 50, 218-227.

39. Yu MW, Robinson FE (1992): The application of shortterm feed restriction to broiler chicken production: a review. J Appl Poult Res, 1, 147-153.

40. Zhan XA, Wang M, Ren H, et al (2007): Effect of early feed restriction on metabolic programming and compensatory growth in broiler chickens. Poult Sci, 86, 654-660.

41. Zubair AK, Leeson S (1996): Compensatory growth in the broiler chicken: a review. World's Poult Sci, 52, 189201.

Geliş tarihi: 06.06.2014 / Kabul tarihi: 18.02.2015
Address for correspondence:
Yrd. Doç. Dr. Özlem Varol Avcllar
Osmaniye Korkut Ata University,
Kadirli School of Applied Sciences Department of Food
Technology,
Kadirli, Osmaniye, Turkey.
e-Mail:ovarol76@gmail.com. 